\title{
Marcadores de estrés oxidativo en placentas de gestantes añosas
}

\author{
Silvia Suárez ${ }^{1}$, Santiago Cabrera ${ }^{2,3}$, Emilio Ramírez ${ }^{4}$, Daysi Janampa ${ }^{4}$
}

Resumen

Palabras clave

\begin{abstract}
Introducción: Un factor de riesgo alto ginecoobstétrico que contribuye a la morbimortalidad materna perinatal es la edad materna mayor de 35 años. Es posible que el estado oxidativo en la placenta de la gestante añosa sea mayor a la de la gestante no añosa. Objetivo: Evaluar el estado oxidativo de la placenta en gestantes mayores de 35 años. Diseño: Estudio observacional y transversal. Lugar: Hospital Nacional Docente Materno Infantil San Bartolomé y Centro de Investigación de Bioquímica y Nutrición de la Universidad Nacional Mayor de San Marcos. Material biológico: 28 placentas de gestantes de 35 años o más y 28 placentas de gestantes de menos de 35 años, obtenidas de partos vaginales a término. Intervenciones: La muestra de placenta se homogenizó al 10\% con buffer fosfato 10 mM, pH 7,4, se centrifugó a 2000 rpm por 5'. Parte del sobrenadante se usó para analizar lipoperoxidación y glutatión (GSH); el sobrenadante restante fue centrifugado a 42000 rpm por 1 hora, para medir actividad de superóxido dismutasa (SOD). Principales medidas de resultados: Estado oxidativo de placentas obtenidas de mujeres añosas y no añosas. Resultados: La actividad de la SOD y el contenido de GSH placentarios en añosas fue 43,52 U/g de tejido y 4,67 $\mu \mathrm{mol} / \mathrm{mL} \times 10^{-2}$; para el grupo control fueron 54,13 $\mathrm{U} / \mathrm{g}$ de tejido $y$ $6,02 \mu \mathrm{mol} / \mathrm{mL} \times 10^{-2}$, respectivamente; en ambos hubo significancia estadística ( $p<$ 0,05). La medición de TBARS (lipoperoxidación) no mostró diferencias significativas. Conclusiones: Las placentas obtenidas de mujeres añosas muestran menor capacidad de defensa antioxidante frente a las placentas obtenidas de mujeres no añosas.
\end{abstract}

Estrés oxidativo; embarazo de alto riesgo; placenta; superóxido dismutasa; glutatión; peroxidación de lípido.

\section{Oxidative stress markers in older pregnant women placentas}

\begin{abstract}
Introduction: A factor for obstetrical high risk that contributes to maternal perinatal morbidity and mortality is maternal age over 35. It is possible that the placenta oxidative state of older women is higher than that of younger pregnant women. Objective: To determine placenta oxidative status in pregnant women 35 years or older. Design: Observational, comparative and crosssectional study. Setting: Saint Bartholomew Mother and Child Teaching Hospital and Biochemistry and Nutrition Research Center, Universidad Nacional Mayor de San
\end{abstract}

1 Centro de Investigación de Bioquímica y Nutrición. Facultad de Medicina, Universidad Nacional Mayor de San Marcos. Lima, Perú.

2 Departamento de Gineco-Obstetricia. Facultad de Medicina, Universidad Nacional Mayor de San Marcos. Lima, Perú.

3 Hospital Docente Materno Infantil San Bartolomé. Lima, Perú.

4 Escuela de Farmacia y Bioquímica. Facultad de Ciencias Biológicas, Universidad Nacional San Cristóbal de Huamanga. Ayacucho, Perú.
Marcos. Biological material: Term vaginal childbirth placentas (28) of women 35 years or older and 28 placentas of pregnant women less than 35 year-old. Interventions: Placenta samples were homogenized to $10 \%$ with buffer phosphate $10 \mathrm{mM}, \mathrm{pH} \mathrm{7,4,} \mathrm{and} \mathrm{centrifuged} \mathrm{to} 2000 \mathrm{rpm}$ by 5'. An aliquot of the supernatant was used to analyze lipoperoxidation and glutathione (GSH); the remaining supernatant was centrifuged to $42000 \mathrm{rpm}$ by 1 hour to determine superoxide dismutase activity (SOD). Main outcome measures: Older and younger mothers' placenta oxidative status. Results: SOD activity and placental GSH content in older women were respectively 43,52 $\mathrm{U} / \mathrm{g}$ of tissue and 4,67 $\times 10^{-2} \mu \mathrm{mol} / \mathrm{mL}$; values for the control group were respectively 54,13 U/g of tissue and 6,02 $\times 10^{-2}$ $\mu \mathrm{mol} / \mathrm{mL}$, both with statistical significant difference $(p<$ 0,05). TBARS (lipoperoxidation) measurements did not show significant differences. Conclusions: Older pregnant placentas showed less antioxidative defence than placentas from younger womens.

Key words: Oxidative stress; pregnancy; high-risk; placenta; superoxide dismutase; glutathione; lipid peroxidation. 


\section{INTRODUCCIÓN}

La morbimortalidad materna representa un importante problema de salud en el país. La edad materna mayor de 35 años (madre añosa) es considerada un factor de riesgo obstétrico alto, así como la diabetes, la multiparidad, la hipertensión arterial (HTA), la presencia de tumores uterinos, etc. Es decir, la edad en una gestante es un factor que predispone a una serie de complicaciones, tales como enfermedad hipertensiva del embarazo (eclampsia, preeclampsia, hipertensión arterial transitoria), abortos, partos prematuros, placenta previa, rotura uterina, atonía uterina, entre otras.

La edad de mayor capacidad biológica ha sido establecida entre 18 y 35 años. A partir de 35 años, el riesgo es mayor, debido a lo que ha sido denominado 'desgaste fisiológico' y a la aparición de patologías propias del envejecimiento $\left({ }^{1}\right)$. En el Perú, como en otros países, la edad mayor a los 35 años se la considera como factor de riesgo de muertes maternas.

En los últimos años se ha detectado que diversos trastornos patológicos están relacionados con especies reactivas del oxígeno (EROs), que pueden llevar a un estado conocido como 'estrés oxidativo'. Este estado tal vez posee un rol importante en el desarrollo de diversas complicaciones presentes en la gestante de 35 años o más.

Dado que el embarazo es un estado fisiológico donde se forman dos productos, la placenta y el feto, se acompaña de una demanda energética elevada y un incremento en los requerimientos de oxígeno. Aún en el embarazo normal se podría esperar un aumento de la carga prooxidante $\left(^{2}\right)$. Durante el desarrollo del periodo gestacional, además del incremento y de la intensa actividad placentaria, se originan también EROs del propio metabolismo embrionario, que pudieran ocasionar bloqueos y restricción del desarrollo $\left(^{3}\right)$.

En la mujer embarazada añosa, debe agregarse además el factor edad que, como se ha mencionado, constituye un factor de riesgo en la morbimortalidad de la madre y el recién nacido. Sin embargo, un estudio epidemiológico previo en nuestro país sobre mortalidad perinatal no evidencia que este factor sea significativo $\left({ }^{4}\right)$.

En la sociedad actual, también es cierto que un grupo de mujeres, por múltiples aspectos económicos, sociales y culturales, posponen la decisión de ser madres. Este grupo sin embargo cuando decide el embarazo lo hace de forma planificada, en términos de nutrición y vigilancia prenatal.

El objetivo del presente trabajo es evaluar el estado oxidativo de la placenta en gestantes mayores de 35 años frente a las gestantes menores de 35 años, con el propósito de contribuir a resolver algunas interrogantes sobre los problemas de morbimortalidad maternoperinatal, que en nuestro país y en Latinoamérica se señala sobre los “35 años como edad límite para el aumento del riesgo para la madre y su perinato" $\left({ }^{5}\right)$.

\section{MÉTODOS}

Se realizó un estudio observacional y transversal utilizándose para ello 56 placentas de embarazos normales obtenidas de partos vaginales a término (36 a 40 semanas), en el Servicio de Gineco-Obstetricia del Hospital Nacional Docente San Bartolomé en Lima (153 msnm), desde agosto del año 2002 a febrero del 2003.

La mitad de dichas placentas correspondió a placentas de mujeres añosas (35 o más años) y 28 placentas para el grupo control (menores de 35 años). Las muestras fueron recogidas e inmediatamente transportadas a $4{ }^{\circ} \mathrm{C}$ al Centro de Investigación de Bioquímica y Nutrición de la Facultad de Medicina de la Universidad Nacional Mayor de San Marcos; fueron almacenadas a $-20^{\circ} \mathrm{C}$, para su análisis posterior.

Para el procesamiento de las muestras se preparó homogenizados al $10 \%$ con buffer fosfato $10 \mathrm{mM}$ a pH 7,4; se centrifugó a $2000 \mathrm{rpm}$ por 5 minutos y se extrajo $3 \mathrm{~mL}$ del sobrenadante para analizar sustancias reactivas al ácido tiobarbitúrico (TBARS, lipoperoxidación), según Buege y Aust $\left({ }^{6}\right)$, como indicador de estrés oxidativo; y glutatión (GSH), por la técnica de Boyne y Ellman $\left({ }^{7}\right)$, como indicador de defensa antioxidante. Lo 
restante se centrifugó a $9000 \mathrm{rpm}$ por 15 minutos y luego a $42000 \mathrm{rpm}$ por 1 hora, para la obtención de citosol y determinación de la actividad de superóxido dismutasa (SOD), como indicador de defensa antioxidante, por el método de Marklund y Marklund $\left({ }^{8}\right)$.

Para el análisis de los datos obtenidos se utilizó el paquete estadístico SPSS 13,0 entorno de Windows. En ambos grupos, la normalidad de los niveles de SOD, GSH, y lipoperoxidación fue comprobada por la prueba de Kolmogorov-Smirnov; luego, se halló el promedio y la desviación estándar, respectivamente. Para comparar las medias poblacionales se usó el $t$ de Student, considerándose una diferencia estadísticamente significativa a una $p<0,05$, para un nivel de significancia del $95 \%$.

\section{RESULTADOS}

Se estudió placentas de embarazos normales obtenidas de partos vaginales a término de madres menores de 35 años, cuyas edades estuvieron en el rango de 20 a 34 años de edad, de las cuales 5 fueron primigestas (18\%) y 23 multigestas $(82 \%)$; asimismo, se estudió las placentas de embarazos normales obtenidas de partos vaginales a término de madres mayores de 35 años, cuyas edades estuvieron en el rango de 35 a 44 años de edad, de las cuales 2 fueron primigestas (7\%) y 26 multigestas $(93 \%)$.

La actividad media de la SOD y el contenido promedio del GSH placentarios en añosas fue 43,52
$\mathrm{U} / \mathrm{g}$ de tejido y $4,67 \mu \mathrm{mol} / \mathrm{mL} \times 10^{-2}$, respectivamente. Para el grupo control, la actividad media de la SOD fue $54,13 \mathrm{U} / \mathrm{g}$ de tejido y el contenido promedio de GSH, $6,02 \mu \mathrm{mol} / \mathrm{mL} \times 10^{-2}$.

En el análisis bioquímico de los indicadores de defensa antioxidante -GSH y SOD- de las muestras de placenta, se encontró que la diferencia entre las medias de ambos grupos de estudio fue estadísticamente significativa $(p<0,05)$.

No se encontró diferencia significativa en ambos grupos de estudio para la evaluación de TBARS como indicador de estrés oxidativo expresado en $\mathrm{nmol} / \mathrm{g}$ de tejido y $\mathrm{nmol} / \mathrm{g}$ de proteína (Tabla 1 ).

\section{DISCUSIÓN}

Las gestantes mayores de 35 años constituyen uno de los grupos de embarazo de riesgo de morbimortalidad de diversa índole, al igual que sus hijos. Salazar y colaboradores $\left(^{1}\right)$ hallaron una mayor incidencia de riesgo de morbimortalidad entre 35 y 38 años. Nuestro estudio precisamente encuentra en ese rango el mayor número de gestantes añosas con embarazo normal a término.

Adicionalmente, en el grupo de las gestantes en estudio, $93 \%$ fue multigesta; aún cuando en el grupo control también predominaron las multigestas, es importante mencionar que además de la edad el hecho de tener varias concepciones conlleva a un desgaste fisiológico que sin un adecuado

Tabla 1. Niveles promedio de indicadores de estrés oxidativo de gestantes mayores e igual a 35 años (añosas) y menores de 35 años.

\begin{tabular}{|c|c|c|c|c|c|}
\hline \multirow[t]{2}{*}{ Indicador } & \multicolumn{2}{|c|}{$\begin{array}{c}\text { Grupo control } \\
n=28\end{array}$} & \multicolumn{2}{|c|}{$\begin{array}{c}\text { Grupo de añosas } \\
\mathrm{n}=28\end{array}$} & \multirow[t]{2}{*}{$p$} \\
\hline & Media & DS & Media & DS & \\
\hline SOD (U/g tejido) & 54,1 & 23.3 & 43,5 & 11,7 & $0,007 *$ \\
\hline GSH (x 10-2 $\mu$ mol$/ \mathrm{mL}$ homog.) & 6,0 & 3,4 & 4,7 & 1,5 & $0,035 *$ \\
\hline TBARS (nmol/g tejido) & 9,4 & 6,1 & 9,6 & 4,1 & 0,443 \\
\hline TBARS (nmol/mg proteína) & 0,3 & 0,2 & 0,3 & 0,1 & 0,326 \\
\hline
\end{tabular}

SOD = Superóxido dismutasa

GSH = Glutation total

TBARS = Lipoperoxidación

${ }^{*} p<0,05$ indica diferencia estadísticamente significativa, por la prueba $t$ de Student 
control de su salud y nutrición pone en riesgo a la gestante $\left({ }^{2}\right)$.

La elevación de la tensión de oxígeno en la sangre materna coincide con cambios morfológicos en las arterias uterinas, que permiten el libre flujo de sangre a la placenta y que provocan alteraciones que se manifiestan por la expresión de la forma inducible de la proteína de shock térmico 70, la formación de residuos de nitrotirosina y la desorganización de las crestas mitocondriales dentro del sincitiotrofoblasto $\left({ }^{9}\right)$, alteraciones que indican que mientras se establece la circulación materna ocurre un 'estallido' oxidativo en la placenta, que pudiera ocasionar los mencionados efectos negativos provocados por los EROs.

Céspedes $\left({ }^{10}\right)$ manifiesta que con una nutrición adecuada rica en antioxidantes se aporta vitaminas y minerales que contribuyen a una buena reserva fisiólogica y por ende a una buena salud, la cual tiene relación con un adecuado balance oxidativo. Igualmente, Gutiérrez $\left({ }^{2}\right)$ propone una nueva estrategia común dirigida al control de los factores que propician el estrés oxidativo, mediante una visión antioxidante de la atención al embarazo.

La actividad significativamente disminuida de la enzima SOD citosólica en placenta de gestantes añosas indica una menor capacidad de dismutación del radical libre superóxido, y por ende uno de los mecanismos enzimáticos antioxidantes disminuido. A este evento se añade también los niveles bajos de GSH total, tripéptido que actúa como metabolito antioxidante endógeno y como cosustrato de otra enzima antioxidante, la glutatión peroxidasa. $\mathrm{Si}$ bien, la forma predominante es la reducida, una disminución del contenido es una señal de agotamiento, tal vez de la síntesis o del sistema de recuperación de esta molécula $\left({ }^{11,12}\right)$.

Son escasos los estudios sobre la implicancia del factor edad de una gestante añosa con respecto a mecanismos antioxidantes. La mayor parte de los estudios en placenta se los enfoca desde el punto de vista patológico. Existen reportes, como el de Clapés, que ha determinado mayor estrés oxidativo en gestantes con diabetes mellitus, patología que se presenta especialmente en gestantes maduras $\left.{ }^{3}\right)$. Asimismo, Vergaray y colaboradores han encontrado que el incremento de la actividad de SOD es reflejo de una elevada generación de anión superóxido en la célula. Sin embargo, una intensa producción de este radical por tiempo prolongado agota la estimulación de la actividad de esta enzima $\left({ }^{13,14}\right)$. Esta sería una explicación de la disminución de la actividad de SOD en las gestantes añosas que encontramos en el presente estudio.

En cuanto al indicador prooxidante TBARS, Perkins manifiesta que la placenta es fuente de los productos de lipoperoxidación durante el embarazo. También, indica que la peroxidación lipídica en el tejido placentario aumenta en función a la edad gestacional $\left({ }^{15}\right)$.

Nuestros resultados no muestran diferencia estadísticamente significativa entre los niveles de TBARS placentario de los grupos comparados. Analizado junto a los otros resultados, puede proponerse que los mecanismos de defensa antioxidante han estado actuando de manera que no se produzca incremento de la peroxidación de lípidos durante el desarrollo de la gestación. Sin embargo, hacia el final del embarazo estas defensas muestran agotamiento o tal vez sea parte del mecanismo fisiológico que conduce a un incremento en la formación del radical superóxido, radical que activa la síntesis de la prostaglandina E2, prostanoide implicado en el inicio de la señal de parto $\left({ }^{16,17}\right)$.

Sin embargo, el hecho de tener menor actividad antioxidante, en lo que se refiere a la SOD y GSH en las gestantes añosas, implicaría un mayor daño oxidativo potencial o daño oxidativo subclínico expresado por otros mecanismos diferentes a la lipoperoxidación. También, puede proponerse que la gestante añosa aún no complicada necesitaría disponer de otros sistemas antioxidantes que compensen a la SOD y GSH disminuidas, para no llegar a producir mayor daño oxidativo $\left({ }^{18}\right)$.

Por otro lado, es necesario enfatizar que las muestras de placentas fueron obtenidas de un Hospital del Ministerio de Salud, donde usualmente acuden mujeres de condición socioeconómica baja, con las características generales de no recibir consejería antes ni después de la concepción, respecto a la nutrición y a los riesgos del embarazo. Sería importante, por lo tanto, evaluar estos mismo pa- 
rámetros en mujeres maduras y profesionales que intencionalmente retardan su embarazo y, cuando lo planifican, se preparan para el inicio de la gestación, llevando un control prenatal adecuado. Este tipo de evaluación se ha descrito en estudios epidemiológicos y sociológicos $\left({ }^{19,20}\right)$ y los resultados merecen un análisis desde diversas perspectivas.

En conclusión, las placentas obtenidas de mujeres añosas muestran menor capacidad de defensa antioxidante frente a las placentas obtenidas de mujeres no añosas, en el presente trabajo. Nuestros hallazgos sugieren que los mecanismos oxidativos actúan de forma diferente en un embarazo en la edad madura (sin patologías asociadas), que en la etapa ideal de gestación, por lo que concordamos con Gutiérrez $\left(^{2}\right)$ en proponer un manejo diferente, mediante una visión antioxidante de la atención al embarazo, que permita disminuir los riesgos de morbimortalidad materna-perinatal.

\section{REFERENCIAS BIBLIOGRÁFICAS}

1. Salazar M, Pacheco J, Scaglia I, Lama J, Munaylla R. La edad materna avanzada como factor de riesgo de morbimortalidad. Ginecol Obstet (Perú). 1999;45(2):124-30.

2. Gutiérrez A. Estrés oxidativo en la gestación: ¿una nueva óptica en la atención a la embarazada?. Rev Cubana Obstet Ginecol. 2005;31(1).

3. Clapés S. Diabetes mellitus, estrés oxidativo y embarazo. Rev Cubana Invest Biomed. 2000;19(3):191-5.

4. Ticona M, Huanco D. Mortalidad perinatal hospitalaria en el Perú: factores de riesgo. Rev Chil Obstet Ginecol. 2005;70(5):313-7.

5. Pacheco J. Ginecología, Obstetricia y Reproducción. Segunda edición. Lima: REP SAC; 2007.

6. Buege JA, Aust SD. Microsomal lipid peroxidation. Methods Enzymology. 1978;52;302-6.

7. Boyne AF, Ellman GL. Assay for glutathione. Anal Biochem. 1972;46;639-53.

8. Marklund S, Marklund G. Involvement of the superoxide anion radical in the autoxidaton of pyrogallol and a convenient assay for superoxide dismutase. Biochem. 1974;47;469-74.

9. Vural P, Akgul C, Yildirim A, Canbaz M. Antioxidant defense in recurrent abortion. Clin Chim Acta. 2000;295(12):169-77.
10. Céspedes.T. y Sánchez, D. Algunos Aspectos sobre el estré oxidativo, el estado antioxidante y la terapia de Suplementación. Revista Cubana Cardiol. 2000;14(1):5560.

11. Mahadik V, Sina A. Study of serum levels of superoxide dismutase in preeclampsia and eclampsia: role of the test as a predictive tool. J Obstet Gynaecol Res. 2003;29(4):262-7.

12. Burton G, Jauniaux E. Placental oxidative stress: from miscarriage to preeclampsia. J Soc Gynecol Invest. 2004;11(6):342-52.

13. Vergaray L, Robles Y, Flores E, Suárez S. Correlación entre los niveles de hemoglobina glicada y las enzimas antioxidantes, en pacientes con diabetes mellitus tipo 2 . Rev Perú Ciencias de Inv. 2000;3:49-56.

14. Walsh SW, Wang Y. Deficient glutathione peroxidase activity in preeclampsia is associated with increased placental production of thromboxane and lipid peroxides. Am J Obstet Gynecol. 1993;169(6):1456-61.

15. Perkins A. Endogenous anti-oxidants in pregnancy and preeclampsia. Austral N Zeal J Obstet Gynaecol. 2006;46(2):77-83.

16. Companioni M. Acido araquidónico y radicales libres: su relación con el proceso inflamatorio. Rev Cubana Invest Bioméd. 1995;14(1):13-7.

17. López Bernal A. Mechanisms of labour-biochemical aspect. Inter J Obstet Gynecol. 2003;110(20):39-45.

18. Gitto E, Reiter RJ, Karbownik M, Tan D, Gitto P, Barberi $\mathrm{S}$, et al. Causes of oxidative stress in the pre- and postnatal period. Biol Neonate. 2002;81(3):146-57.

19. Salihu H, Shumpert N, Slay M, Kirby R, Alexander G. Childbearing beyond maternal age 50 and fetal outcomes in the United States. Obstet Gynecol. 2003;102:1006-14.

20. Olivar A. Cambios biológicos, psicológicos y sociales durante el embarazo. Rev Fed Odont Colomb. 2002;201:37-51.

Manuscrito recibido el 17 de octubre de 2007 y aceptado para publicación el 13 de diciembre de 2007.

\author{
Correspondencia: \\ Mg. Silvia Suarez Cunza \\ Centro de Investigación de Bioquímica y Nutrición \\ Facultad de Medicina - UNMSM \\ Av. Grau 755. Lima 1, Perú \\ Correo-e: ssuarezc@unmsm.edu.pe
}

